\section{ESTUDIO DE LOS PATRONES DE CONDUCTA DEL ALUMNADO CON TDAH EN LA CLASE DE EDUCACIÓN FÍSICA}

\author{
ESTUDO DOS MODELOS DE COMPORTAMENTO DO ESTUDANTE COM TDAH \\ NA CLASSE DE EDUCAÇÃO FÍSICA
}

\author{
ADHD BEHAVIOR PATTERNS IN PHYSICAL EDUCATION CLASSES IN \\ PRIMARY SCHOOL
}

\author{
Víctor Labrador Roca*, Francisco Javier Hernández Vázquez*, \\ Eduard Inglés Yuba*
}

\begin{abstract}
Palabras clave: Trastorno por Déficit de Atención con Hiperactividad. Educación y Entrenamiento Físico.

Observación Entrevista.

Resumen: El objetivo del trabajo fue identificar los patrones de conducta del alumnado diagnosticado con TDAH en las clases de Educación Física (EF) en las escuelas de Educación Primaria de Barcelona (España). Seis estudiantes y sus docentes de EF fueron observados durante 42 sesiones completas. Los maestros fueron entrevistados después del conjunto de observaciones durante un tiempo promedio de 65 minutos. El alumnado con TDAH mostró tres categorías de conductas deseables y cuatro categorías de conductas no deseables. El $18,65 \%$ de las conductas registradas fueron deseables y el $81,35 \%$ no deseables. Los resultados sugieren que el profesorado debería centrarse no solo en las dificultades planteadas por el trastorno (es decir, conductas no deseables), sino que deberían mejorar los aspectos positivos de los estudiantes con TDAH. La forma de administrar la clase y las actividades deportivas y el fomento de actividades creativas mejoran las conductas deseables en las sesiones de EF.
\end{abstract}

Palavras chave: Transtorno do Déficit de Atenção com Hiperatividade. Educação Física e Treinamento. Observação. Entrevista.

Keywords: Attention Deficit/ Hyperactivity Disorder. Physical Education and Training. Observation Interview.

Resumo: 0 objetivo foi identificar os padrões de comportamento em aulas de Educação Física (EF) de alunos com diagnóstico de TDAH nas escolas primárias de Barcelona (Espanha). Seis estudantes e seus seis professores de Educação Física foram observados durante 42 aulas inteiras de Educação Física. Os professores foram entrevistados após o conjunto de observações por um tempo médio de 65 minutos. Os alunos com TDAH mostraram três categorias de comportamentos desejáveis e quatro categorias de comportamentos indesejáveis; $18,65 \%$ dos comportamentos registrados foram desejáveis e $81,35 \%$ indesejáveis. Os resultados sugerem que os professores devem se concentrar não apenas nas dificuldades colocadas pelo transtorno (ou seja, comportamentos indesejáveis), mas devem melhorar os aspectos positivos dos alunos com TDAH. A maneira de gerenciar a classe, o tipo de jogos, atividades esportivas e incentivar atividades criativas, aumentar os comportamentos desejáveis nas sessões de PE.

Abstract: The purpose of the study was to identify the behavioral patterns of students diagnosed with ADHD in Physical Education (PE) classes in primary schools of Barcelona, Spain. Six students and their PE teachers were observed during 42 entire lessons. After the observations, teachers were interviewed for 65 minutes on average. Students with ADHD displayed three categories of desirable behavior and four categories of undesirable behavior; $18.65 \%$ of behaviors registered were desirable and $81.35 \%$ were undesirable. The results suggest that teachers should not only focus on the difficulties posed by the disorder (i.e., undesirable behaviors), but also enhance the positive aspects of students with ADHD. The way to manage sections, give sports lessons and encourage creative activities enhance desirable behaviors in PE lessons.
*Universitat de Barcelona. Barcelona, Espanha.

E-mail: vilaroca86@gmail.com; jhernand@gencat.cat; eduard.ingles@gencat.cat

Recebido em: 10-04-2018 Aprovado em: 29-04-2019 Publicado em: 25-06-2019

$\mathrm{DOl}$ https://doi.org/10.22456/1982-8918.81877 (c) (1) (8) Licence 


\section{INTRODUCCIÓN}

El trastorno por déficit de atención con hiperactividad (TDAH) se considera un trastorno neuroconductual comúnmente diagnosticado durante la infancia, pero la frecuencia de la afección no es ampliamente reconocida en muchos países (GOULARDINS; MARQUES; OLIVEIRA, 2017). Por esta razón y con objeto de profundizar en el estudio del comportamiento en alumnado diagnosticado con TDAH, el objetivo de este estudio fue identificar los patrones de conducta del alumnado diagnosticado con TDAH durante las clases de Educación Física (EF) en seis centros educativos de Barcelona (España) durante todo un curso escolar. Para ello, fue necesario combinar el conocimiento científico que se analiza a continuación y crear una tabla de contenidos que enumerara las conductas de interés.

La versión del Manual Diagnóstico y Estadístico de los Trastornos Mentales $4^{\underline{a}}$ edición revisada (DSM-IV-TR) utilizada entre 2002 y 2013 y la nueva versión (DSM-V) utilizada a partir del año 2013, describe diversas características del TDAH. De forma más concreta, este trastorno se define como un patrón persistente de falta de atención y / 0 hiperactividad-impulsividad, más frecuente y grave de lo que se observa en individuos con un nivel de desarrollo similar (AMERICAN PSYCHIATRIC ASSOCIATION, 2002). Las conductas manifiestas incluyen trastornos del comportamiento, trastornos del sueño, falta de autocontrol, agresión, baja coordinación motriz, depresión, ansiedad o falta de respeto por las reglas del juego (CARRIEDO, 2014).

En España, se ha confirmado que el 6,8\% de los estudiantes de entre 5 y 15 años están diagnosticados con TDAH (CATALÁ-LÓPEZ et al., 2012). En este sentido, la APA (2013) afirma que no existe un consenso global sobre la prevalencia del TDAH en niños, ya que los análisis de metarregresión han estimado la prevalencia mundial entre 5,29\% y 7,1\% (POLANCZYK; WILLCUTT; SALUM; KIELING; ROHDE, 2014).

El TDAH se ha estudiado desde perspectivas médicas y psicológicas, pero en España al menos, el aspecto educativo ha sido ignorado en gran medida (JIN; LI, DU; COGHILL; AU; ZHONG, 2016). Los estudiantes con TDAH también tienen trastornos comórbidos, como: problemas afectivos (trastornos del estado de ánimo o ansiedad), trastornos del aprendizaje, fracaso escolar, trastornos del comportamiento y, cada vez más, trastornos de coordinación del desarrollo (GUSTAFSSON et al., 2010). A pesar de la alta prevalencia del TDAH en el contexto educativo, diversos autores señalan que los maestros carecen de conocimiento sobre el trastorno (FEU et al., 2016; TANURE ALVES et al., 2017). Además, "[...] aunque es común que las maestrías en educación física se gradúen con conocimientos y habilidades basadas en principios científicos, muchas no desarrollan la capacidad de manejar conductas problemáticas de los estudiantes" (LAVAY; HENDERSON; FRENCH; GUTHRIE, 2012, p. 195).

En este estudio se distinguen dos tipos de conductas en estudiantes con TDAH en el contexto de la EF: conductas deseables y conductas no deseables. Obviamente, para definir las "conductas deseables" debemos indagar sobre las posibles fronteras entre "no deseable" y "deseable" o, tal y como cita Moreno Oliver (2001), los límites entre lo "no problemático" y lo "problemático". Este autor, define el comportamiento no deseable como el comportamiento excesivo e inapropiado que causa problemas.

En sentido contrario, McGinnis y Goldstein (1990) describen un conjunto de habilidades diseñadas para reforzar el desarrollo prosocial de preescolares y escolares. Este conjunto 
de habilidades es similar al conjunto de "conductas deseables" para los estudiantes en la escuela mencionados anteriormente. Además, en la publicación Estrategias para mejorar el comportamiento y el rendimiento escolar de los niños con TDAH en el aula, Orjales (2013) divide un conjunto de conductas en diferentes categorías también tenidas en cuenta para la identificación de los patrones de conducta del alumnado con TDAH en este estudio.

Otra herramienta que puede proporcionar información sobre el tipo de conductas del alumnado con TDAH en clases de EF es la Escala de Calificación revisada de Conners (CRS-R), una herramienta reconocida que proporciona una evaluación integral del TDAH y condiciones comórbidas como trastorno oposicional desafiante y trastornos de conducta. En 2015, se publicó Conners 3rd Edition TM después de cuatro años de investigación y desarrollo de productos creados para satisfacer las demandas de psicólogos escolares, médicos, psiquiatras, pediatras, agencias de protección infantil y trabajadores de la salud mental. Entre las conductas más comunes, la escala de Conners incluye la psicopatología general; inatención; hiperactividad / impulsividad; dificultades de aprendizaje; funcionamiento ejecutivo; desafío I agresión; relaciones de compañeros; y trastornos del comportamiento (WYK; PIENAAR; COETZEE, 2012).

Otro ejemplo del área de EF se menciona en el artículo de Aragón Calvo (2011) quien crea un instrumento de observación para identificar los comportamientos habituales de los estudiantes con TDAH en las clases de EF. Esta herramienta incluye las siguientes categorías: falta de atención, hiperactividad, impulsividad y otras situaciones (gritos, golpes, golpes, insultos, reacciones excesivas, aburrimiento, etc.).

En la misma línea, la clasificación del comportamiento no deseable se presenta en términos de los elementos derivados del estudio de campo de Efstratopoulou, Janssen y Simons (2012) quienes clasifican las categorías de comportamiento no deseable como: desobediencia, agresión, hiperactividad, impulsividad, falta de atención, aislamiento y ansiedad.

\section{MÉTODO}

\subsection{PARTICIPANTES}

Este estudio se realizó en seis escuelas de educación primaria, públicas de la ciudad de Barcelona (España). El alumnado con TDAH era elegible si cumplía los siguientes criterios: (a) diagnóstico clínico de TDAH realizado por el médico del niño, (b) un niño en cuarto, quinto o sexto grado (9, 10 o 11 años) y (c) deberían tomar medicamentos recetados a primera hora de la mañana (específicamente el clorhidrato de metilfenidato: este tratamiento es común en personas con TDAH de subtipo combinado). El alumnado con TDAH fue diagnosticado por escrito por el médico del niño y fueron confirmados por el informe Conners 3-Parent, donde el $95 \%$ de los estudiantes obtuvieron puntuaciones en el rango clínico de 65 o más en el TDAH DSM-IV hiperactivo / impulsivo.

Además, sus respectivos docentes de EF (edad en años: $M=40.33, S D=4.85$ ) participaron en el estudio. Todos tenían más de cinco años de experiencia docente $(M=15, S D$ = 6.38). El alumnado con TDAH (edad en años: $M=9.50, D E=1.12$ ) fue diagnosticado usando los criterios del DSM-IV (AMERICAN PSYCHIATRIC ASSOCIATION, 2002). Fueron evaluados 
individualmente para validar el diagnóstico preliminar y especificar el diagnóstico diferencial. Todos tenían el subtipo combinado (hiperactividad, impulsividad y comportamiento de déficit de atención), basado en el código de tipo 314.01 (AMERICAN PSYCHIATRIC ASSOCIATION, 2002), y todos recibían medicación durante todo el estudio. Los métodos de recopilación de datos incluyeron: (a) observación en el aula y (b) entrevistas en profundidad. Por lo tanto, los investigadores utilizaron una variedad de fuentes de datos como medio de triangulación (MERRIAM, 1998). Todas las entrevistas y todas las observaciones se registraron con el consentimiento de los informantes y los padres, madres o tutores legales de los estudiantes.

\subsection{FASE I: OBSERVACIÓN}

Para estudiar el comportamiento de los estudiantes en edad escolar, Efstratopoulou, Janssen y Simons (2012) propusieron observar al niño en diferentes contextos y situaciones como el método más válido y confiable para establecer cómo interactúan con los docentes y con sus compañeros, y cómo juegan, comparten y obedecen, etc. En este estudio, los estudiantes fueron observados durante 42 sesiones completas de EF de una hora de duración durante un año académico (con un promedio de siete sesiones en cada escuela).

Los investigadores adoptaron el papel de observadores no participantes basados en las descripciones de Abikoff et al. (2002), ya que los investigadores siempre mantuvieron el papel de observadores externos y se mantuvieron a una distancia suficiente para evitar involucrarse en las sesiones y, en la medida de lo posible, evitar distorsionar la dinámica de las sesiones. Además, en vista de la posición de los investigadores, se decidió utilizar la observación encubierta (SOMMER; SOMMER, 2002); los estudiantes conocían el tema general del estudio (para evaluar su comportamiento en las clases de EF), pero no los detalles específicos (si la conducta era deseable o no deseable en relación con las diferentes variables). Finalmente, el protocolo de observación comprendió una observación estandarizada basada en el uso de notas de campo y registro narrativo, que contiene una serie de descripciones y reflexiones recibidas y registradas por el investigador en el contexto natural (LATORRE, 2007).

Los datos de las observaciones se recolectaron usando una grabadora de audio para ayudar al investigador a generar una "descripción detallada" (CARSPECKEN, 1999, p. 54). Además, las notas de campo fueron escritas consecutivamente durante el período de observación por el investigador cada vez que sucedía algo durante la sesión. Luego, el investigador revisó los audios grabados de las observaciones con las notas de campo tomadas durante las observaciones después de cada sesión. Toda la información fue escrita por el investigador en una hoja de datos para facilitar la futura tarea de descripción.

Anteriormente, para probar el instrumento de investigación de observación, dos observadores registraron de forma independiente a los mismos estudiantes durante la misma cantidad de sesiones. El acuerdo Interobservador (IOA) se registró en 16 sesiones de EF anteriores a la investigación oficial. Cada observador tuvo que aplicar las categorías preestablecidas, con base a un conjunto de conductas de estudiantes con TDAH encontrados en la búsqueda bibliográfica y especificados durante la prueba piloto. El IOA se calculó dividiendo el número de conductas acordadas por el número total de conductas y multiplicando el resultado por 100 (COOPER; HERON; HEWARD, 2007). El promedio de IOA en todos los estudiantes fue del $85 \%$ (rango $80 \%$ - 90\%), para la clase de EF y del $87 \%$ (rango $85 \%$ - 90\%) 
para el periodo de recreo. Cooper, Heron y Heward (2007) recomiendan un promedio de $80 \%$ cuando múltiples comportamientos son evaluados por dos o más observadores.

Se observaron 42 sesiones de EF en seis escuelas diferentes $(M=7, D E=2,31)$ para describir las conductas del alumnado con TDAH. La observación, basada en los indicadores de la Tabla 1 y 2 fue probada y validada durante la prueba piloto, y se usó como referencia durante el trabajo de campo.

\subsection{FASE II: ENTREVISTA AL PROFESORADO DE EDUCACIÓN FÍSICA}

Posteriormente, se entrevistó al profesorado de EF para completar la información recopilada durante la observación. Se aplicaron análisis descriptivos e inferenciales a los datos cuantitativos, y los datos cualitativos se sometieron a análisis de contenido de los registros narrativos.

La entrevista comprendía preguntas planteadas en el contexto de la investigación para obtener información de las personas en estudio que podrían ayudar a resolver la cuestión central de la investigación. Las entrevistas no fueron directivas y comenzaron con una sola declaración como invitación: "Hablemos del comportamiento del alumnado con TDAH en las clases de EF". Las entrevistas duraron un promedio de 65 minutos.

Para las entrevistas en profundidad, se desarrolló un protocolo de entrevista basado en la literatura existente (KIELY; ASKHAM, 2012) para alentar al profesorado a describir y evaluar sus propias experiencias de aprendizaje completa y libremente desde su propia perspectiva. Todas las entrevistas se llevaron a cabo cara a cara y se grabaron con audio para fines de transcripción. Las entrevistas se llevaron a cabo en la misma escuela de los estudiantes observados. Las entrevistas se transcribieron inmediatamente y el investigador revisó cada transcripción con notas escritas de la entrevista mientras escuchaba la grabación correspondiente.

Debido a la naturaleza y propósitos de este estudio, el método de investigación adoptado es uno de los más importantes en el desarrollo de las ciencias sociales y uno de los más utilizados en la investigación en EF: el estudio de caso (SOLER PRAT; VILANOVA SOLER, 2010). Todos los procedimientos estadísticos se analizaron con el paquete estadístico 'Microsoft Excel 2010' para Windows. Y todos los textos fueron insertados en el programa 'Nudist Nvivo@, para codificar todas las entrevistas del profesorado de EF.

\section{RESULTADOS}

\subsection{FASE I: CONDUCTAS DEL ALUMNADO CON TDAH EN LAS SESIONES DE EDUCACIÓN FÍSICA OBSERVADAS}

Se observaron un total de 681 conductas en las 42 sesiones de EF. La cantidad de conductas registradas por sesión varió, con un promedio de 16.21 (DE = 7.15). Con base en estas grabaciones, se observaron 43 conductas diferentes; tanto deseables como no deseables (Tabla 1 y 2 ).

El análisis de las observaciones de los investigadores reveló que 17 de los 43 indicadores (39.53\%) podían clasificarse en tres categorías (variables) de conductas deseables: 
habilidades sociales básicas y manejo de los sentimientos; habilidades relacionadas con el maestro; y habilidades relacionadas con los compañeros. Los otros 26 indicadores (60.47\%) reflejaron conductas no deseables: hiperactividad, impulsividad, déficits de atención y otros comportamientos (desobediencia, gritos, berrinches, búsqueda de atención, comportamiento perturbador con otros y aburrimiento).

Uno de los resultados más llamativos fue el hecho de que el 19\% de las formas de comportamiento se consideraron conductas deseables (tabla 1), y el $81 \%$ restante se percibieron como no deseables (Tabla 2). Una vez que se identificaron las conductas deseables de los estudiantes con TDAH a través de la observación, se resaltaron los comportamientos más comunes. En la variable "habilidades sociales y gestión de los sentimientos", el "esfuerzo para cumplir con las instrucciones del docente" $(5,29 \%)$ fue la conducta más observada. Entre las "habilidades relacionadas con el maestro", "ayudar al maestro" fue la más frecuente $(0,44 \%)$. Y finalmente, en la variable "habilidades relacionadas con los compañeros", el indicador registrado con mayor frecuencia fue "ayudar a los compañeros" (3,23\%).

Tabla 1 - Registro de las conductas deseables de alumnado con TDAH.

\begin{tabular}{|c|c|c|c|}
\hline Dimensiones & Variables & Indicadores & $\begin{array}{l}\% \text { de } \\
\text { registros }\end{array}$ \\
\hline \multirow{17}{*}{$\begin{array}{l}\text { Conductas } \\
\text { deseables }\end{array}$} & \multirow{8}{*}{$\begin{array}{l}\text { Habilidades sociales } \\
\text { básicas y manejo de } \\
\text { sentimientos }\end{array}$} & Aceptación de posibilidades y limitaciones & 0.15 \\
\hline & & Aceptación de responsabilidades & 1.32 \\
\hline & & Gratitud & 0.15 \\
\hline & & Esfuerzo para cumplir con las instrucciones del docente & 5.29 \\
\hline & & Disfrute & 2.06 \\
\hline & & Optimismo & 1.03 \\
\hline & & Simpatía & 0.88 \\
\hline & & Auto confianza & 0.44 \\
\hline & \multirow{3}{*}{$\begin{array}{l}\text { Habilidades } \\
\text { relacionadas con el } \\
\text { profesorado de EF }\end{array}$} & Respeto por las decisiones & 0.29 \\
\hline & & Ayuda al profesorado & 0.44 \\
\hline & & Atención a los comentarios del profesorado & 0.15 \\
\hline & \multirow{6}{*}{$\begin{array}{l}\text { Habilidades } \\
\text { relacionadas con los } \\
\text { compañeros }\end{array}$} & Interés en lo que le sucede a los compañeros & 0.73 \\
\hline & & Respetando su turno & 1.62 \\
\hline & & Ayudando a los compañeros & 3.23 \\
\hline & & Compartir cosas & 0.29 \\
\hline & & $\begin{array}{l}\text { Persuadir a otros a través de la razón, no a través de } \\
\text { gritos o imposiciones }\end{array}$ & 0.15 \\
\hline & & Buenas relaciones con los compañeros & 0.59 \\
\hline TOTAL & & & 19.54 \\
\hline
\end{tabular}

Al dividir las conductas no deseables en sus respectivas variables (tabla 2), las conductas hiperactivas más comunes fueron "aburrirse durante periodos de espera" (5,58\%) y "hacer cosas peligrosas" (3,67\%); el comportamiento impulsivo más frecuente fue "interrumpir o expresar una opinión fuera de turno" (11.01\%). Estos resultados fueron corroborados en entrevistas por casi todo el profesorado de EF. Entre las conductas de déficit de atención, 
la más frecuente fue "no escuchar» $(6,02 \%)$, y finalmente, en otras conductas, "no cuida el material deportivo" (4,11\%), "requiere atención" (11,16\%) y "se enfada" fueron las conductas más comunes $(5.29 \%)$.

Tabla 2 - Registro de las conductas no deseables de alumnado con TDAH.

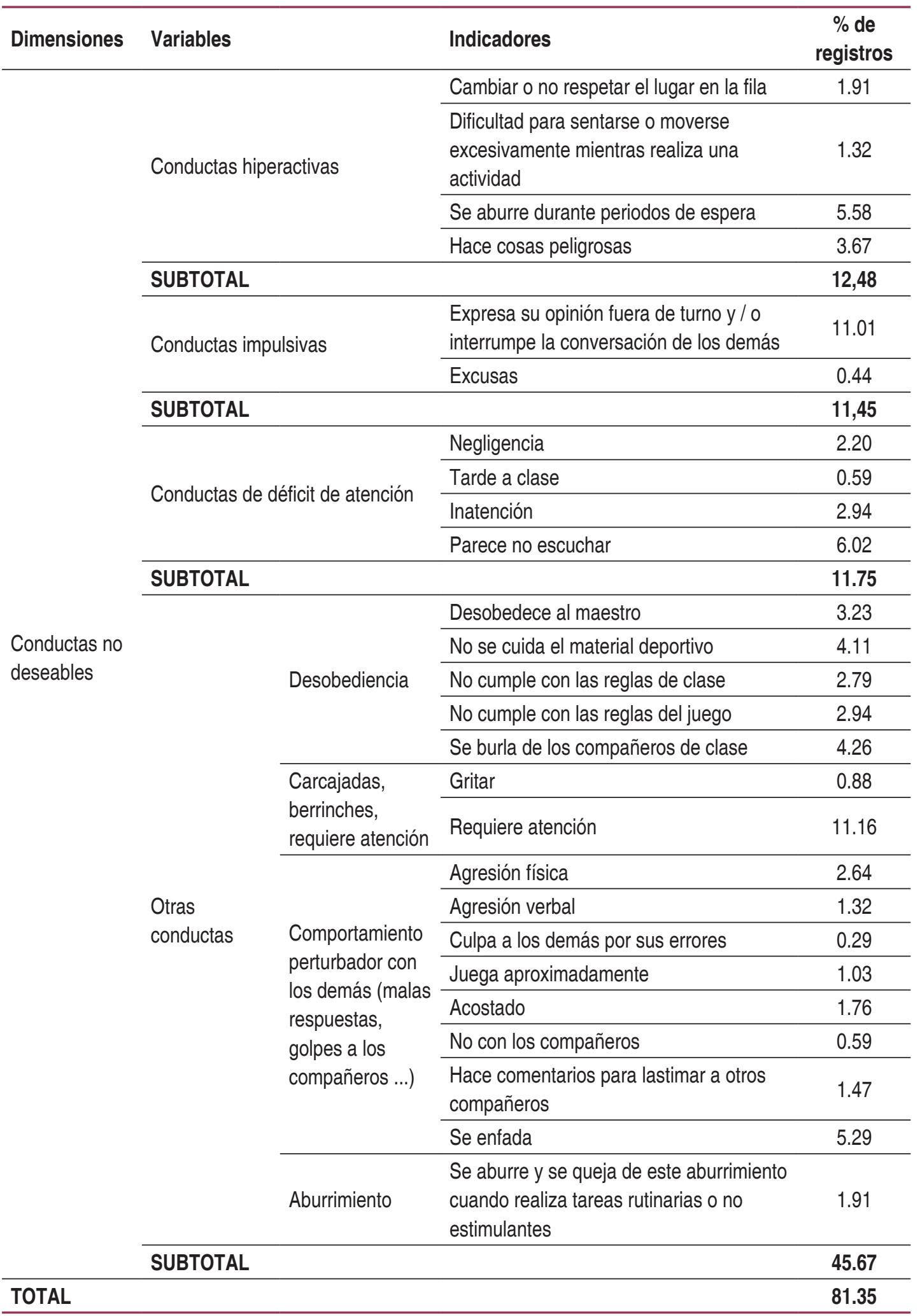

Fuente: Elaboración propia 


\subsection{FASE II: CONDUCTAS DEL ALUMNADO CON TDAH EN LAS CLASES DE EF CON BASE EN LAS ENTREVISTAS}

Después del periodo de observación, para identificar cómo el conjunto docente describía las conductas del alumnado con TDAH en las clases de EF, se entrevistó al profesorado de EF quien no conocía los resultados de las observaciones realizadas. Así pues, durante el periodo de observación, los docentes solo sabían que el estudio involucraba el análisis de los estudiantes con TDAH y no sabían que iban a ser entrevistados después de la observación. Significativamente, el conjunto de profesorado entrevistado habló sobre algunos de los indicadores obtenidos en la Fase I (observación).

Sus descripciones fueron muy similares en contenido a las observaciones. Por ejemplo, cuando hablaban sobre la conducta deseable, específicamente la aceptación de responsabilidades, algunos de los maestros comentaron "quizás él / ella tenga un sentido altamente desarrollado de justicia y aplique las reglas del juego para todos". Con respecto al "esfuerzo para cumplir con las instrucciones", algunas de las afirmaciones fueron en la línea de "le gusta competir con aquellos que son tan buenos como él". En cuestiones de habilidades, "él / ella se esfuerza". Otros comportamientos comentados por los profesores fueron la "simpatía" y la "confianza en sí mismos".

Muchos docentes también hablaban que el alumnado con TDAH siempre ofrece ayuda: "es uno de los que siempre quiere ayudarme". Siguiendo con las conductas deseables mencionan: "sobre ayudar al profesorado, le gusta ayudar, guardando cosas, arreglando el gimnasio".

Curiosamente, durante la entrevista, el conjunto docente no mencionó las siguientes conductas deseables: "aceptación de las posibilidades y limitaciones", "gratitud", "disfrute", "optimismo", "respeto por las decisiones", "atención a los comentarios y explicaciones del profesorado", "interés en lo que les sucede a los compañeros", "respeto del turno de otras personas", "ayudar a los compañeros de clase", "compartir material" y "persuadir a otros a través de la razón en lugar de gritar o imponer". Sin embargo, todas estas conductas deseables se habían registrado durante el período de observación.

Cuando el profesorado describía las conductas no deseables, mencionaron "cambiar o no respetar el lugar en la fila". Algunos de ellos dijeron "[...] él / ella no suele respetar la fila". Por ejemplo, "cuando voy a clase, cuando los estudiantes están fuera de clase, por lo general se ponen en fila. Algunos de ellos tienen dificultades para estar en la fila, no paran de moverse, conversar, [...]". Varios maestros percibían la dificultad que tenían los estudiantes para esperar pacientemente una explicación o para completar un ejercicio en particular. Otra conducta frecuente no deseable es cuando el alumnado con TDAH molesta a sus compañeros durante los períodos de descanso o pausas. Los maestros comentaron: "[...] las pausas o los períodos de descanso son terribles [...] molesta a otra persona o intenta jugar con alguien que no está interesado en jugar con él".

Otra conducta no deseable mencionada es la "toma de turnos inesperada" o la "interrupción de otros". Los docentes informaron: "[...] no respeta nada ... no sabe lo que significa levantar la mano ... solo habla directamente". La búsqueda de atención era otra conducta común del alumnado con TDAH. Todos los maestros expresaron respuestas como: 
"[...] quiere que todos lo escuchen ... exagera el dolor ... y el / ella finge que se ha roto la pierna". Con respecto a la conducta provocativa, un docente dijo que era complicado saber hasta qué punto el alumnado lo hacía de forma consciente o inconsciente.

Los docentes comentaron varios temas relacionados con la negligencia que manifiestan los estudiantes observados. Por ejemplo: "[...] es el último en abandonar la clase". "Y él / ella siempre es el último: porque se queda hurgando en un cajón, colocando las cosas dentro de su mochila ...". Muchos docentes corroboraron la alta prevalencia del comportamiento de búsqueda de atención en sus sesiones de EF. Por ejemplo: "[...] a veces exagera un dolor ... o cuando están jugando a un juego, o cuando están haciendo una actividad que requiere un poco más de contacto ... si no juegan de la manera que él quiere ... o su equipo está perdiendo, muestra frustración ... o exagera o se cae sin siquiera ser tocado [...]".

Cuando el alumnado con TDAH se enfada, "[...] es difícil hacerles entender ... que a veces ganas y a veces pierdes ... con él / ella ... tiene dificultades para aceptar la derrota ... y a menudo se enfada". Durante las entrevistas, el profesorado mencionó otras conductas no deseables como, por ejemplo: "[...] no cumplir con las reglas de la clase".

\section{DISCUSIÓN}

El principal hallazgo de esta investigación fue que el alumnado con TDAH en el contexto de la clase de EF muestra dos tipos de comportamiento: conductas deseables y conductas no deseables. Esta diferencia permite a los investigadores observar ambos tipos de conductas y distinguirlas en deseables y no deseables registradas durante el período de observación y mencionadas en las entrevistas con los docentes que participaron en este estudio.

El diagnóstico de TDAH en estos estudiantes se basa en los criterios del DSM-IVTR (AMERICAN PSYCHIATRIC ASSOCIATION , 2002). El manual DSM-IV-TR incluye este trastorno en la sección "trastornos que se inician en la infancia y la adolescencia", y más específicamente en el grupo de "trastornos por déficit de atención y conducta disruptiva", junto con el trastorno de conducta y el trastorno oposicionista / desafiante. Este documento revela una concepción y una atribución de la conducta no deseable o problemática del alumnado diagnosticado con TDAH.

Para discutir, contribuir u oponerse a los resultados de investigaciones previas, en este trabajo se ha revisado varios estudios que analizan el comportamiento del alumnado con TDAH en el contexto escolar, y específicamente en el área de EF (ARAGÓN CALVO, 2011; CARRIEDO, 2014; EFSTRATOPOULOU; JANSSEN; SIMONS, 2012; ORJALES, 2013). Estos estudios revelan una tendencia a enfocarse solamente en conductas no deseables 0 problemáticas del alumnado con TDAH y a descuidar el buen comportamiento o las conductas deseables que pueden aparecer durante las clases de EF por parte de este alumnado.

Es cierto que un gran número de estudiantes con TDAH tiene dificultades para regular su comportamiento y cumplir con los estándares esperados para su edad. De hecho, de acuerdo con Anderson et al. (2012) los estudiantes con TDAH manifiestan dificultades en el contexto familiar, en el entorno escolar y en las relaciones con los compañeros. Este estudio corrobora esta afirmación en la medida en que presenta bajos porcentajes de registros referidos a la capacidad de hacer amigos (una variable incluida en el estudio) y altas tasas de registros 0 comentarios en las entrevistas de los docentes que se refieren al alumnado con TDAH que 
interrumpe a su profesorado o compañeros, manifestando enfado y molestia a sus compañeros de clase.

Coincidiendo con los hallazgos de Barkley (2006), quien señaló que las limitaciones en las habilidades sociales de los estudiantes con TDAH están asociadas con una predisposición del trastorno en sí, en este estudio observamos tasas mucho más bajas de conductas deseables (incluidas las habilidades sociales) que de conductas no deseables. Sin embargo, hacemos hincapié en que el alumnado con TDAH también presenta un buen comportamiento durante las clases de EF, y que estas conductas deben evaluarse y reforzarse.

Este estudio registró comportamientos en relación con las habilidades sociales básicas y el manejo de los sentimientos de los estudiantes con TDAH. El volumen de registros 0 consideraciones hechas por el profesorado participante en el estudio respaldan la afirmación de Barkley (2006) que las "deficiencias en las habilidades sociales características del TDAH no necesariamente indican que los sujetos no pueden desarrollar estas habilidades [...] pero sugieren que los sujetos están limitados por la condición biológica del TDAH” (p. 103).

El estudio de Ashwood et al. (2015) establece una relación entre los estilos cognitivos y las habilidades de adaptación, entendida como el conjunto de habilidades positivas o deseables que un niño puede desarrollar. El mismo estudio concluye sobre la importancia de fortalecer las habilidades sociales en las personas diagnosticadas con TDAH, destacando conductas que coinciden con las conductas descritas en el estudio actual, tales como autoestima, inclusión, expresión de sentimientos, actitudes, opiniones, respeto por los demás, etc.

Esta investigación coincide con la declaración de Mautone, Lefler y Power (2011) que considera esencial fortalecer las habilidades sociales de los estudiantes con TDAH, porque sus dificultades para interactuar con sus compañeros pueden causarle aislamiento del grupo. Además de las habilidades sociales básicas y la gestión de los sentimientos, de acuerdo con McGinnis y Goldstein (1990) este estudio observó otros comportamientos positivos en estudiantes con TDAH, como el esfuerzo por cumplir con las instrucciones del docente, el disfrute y la responsabilidad. En menor medida, también presentaron optimismo y amabilidad.

Barkley (2006) señala el tipo de comportamientos que pueden ocurrir en los estudiantes con TDAH, por ejemplo, una intensa sensación de bienestar, estado de ánimo elevado o euforia, e incluso la sensación de que saben más que otros o tienen habilidades especiales. Los resultados de este estudio reflejan que los estudiantes con TDAH manifiestan simpatía, disfrute, optimismo y confianza en sí mismos.

Después de reconocer la baja, pero significativa afluencia de conductas deseables por parte del alumnado con TDAH en EF, ahora recurrimos a las conductas no deseables, que se registran con mayor frecuencia. La descripción de Tannock (2013) del comportamiento de los estudiantes con TDAH incluye conceptos tales como impaciencia, impulsividad, despreocupación por lo que se les dice, una tendencia a distraer a los demás, problemas para llevarse bien con sus compañeros o dificultad para sentarse cuando es necesario. Este estudio corrobora estos hallazgos, porque durante el transcurso de varias observaciones y entrevistas con docentes, se demostraron comportamientos similares en los estudiantes: cambiar o no respetar su lugar en la fila, dificultad para sentarse cuando sea necesario, realización de acciones peligrosas, interrumpir a otros o expresando una opinión fuera de turno, falta de atención, ignorar lo que se les dice, y desobediencia. 
Además, Van den Ban et al. (2014) coinciden en que la presencia de comportamientos asociados con la hiperactividad ha demostrado ser el principal determinante del TDAH. En este estudio y coincidiendo con Bunford, Evans y Wymbs (2015) uno de los comportamientos más frecuentemente registrados fue la tendencia a molestar a otros estudiantes. También coincidiendo con Orjales (2013) registramos una alta tasa de comportamiento infantil entre los estudiantes con TDAH durante las clases de EF, con el fin de atraer la atención del maestro 0 de sus compañeros al gritar, hacer berrinches o hacer comentarios con voz infantil.

Paralelamente a los comportamientos registrados en relación con la hiperactividad, impulsividad y déficit de atención, que se consideran categorías diferenciales en TDAH, esta investigación también registró una conducta clasificada por Aragón Calvo (2011) bajo el título "otras conductas" como desobediencia, golpeos, comportamiento perturbador con otros y aburrimiento, que son variables importantes que quedan fuera de los tres principales variables del TDAH. Estas descripciones también se incluyen en el estudio de Efstratopoulou Janssen y Simons (2012) quienes crearon estas categorías basadas en entrevistas a profesorado de EF y otros criterios diagnósticos que diferencian entre agresión, aislamiento y ansiedad.

Finalmente, coincidiendo con Friedrichs, Igl, Larsson y Larsson (2010) destacamos la importancia no solo de definir las dificultades del trastorno, sino de centrarnos en los aspectos positivos del alumnado con TDAH. Esto es particularmente así en el caso del subtipo combinado, el grupo que presenta la mayoría de las deficiencias y problemas.

\section{CONCLUSIONES}

Al ubicar este estudio en el contexto de las clases de EF, el propósito es proporcionar una fuente de información específica en el área de la enseñanza de la actividad física inclusiva. La combinación de metodologías cuantitativas y cualitativas (observación y entrevista) ha permitido identificar, describir y analizar la información reunida de una manera más rica y profunda.

En este estudio, se observa que las formas deseables de comportamiento por parte de los estudiantes con TDAH rara vez son registradas por el profesorado. Esta investigación ha revelado un porcentaje mucho mayor de formas de conducta no deseable que de conducta deseable. Una conclusión es que el conjunto docente debe esforzarse por crear situaciones que fomenten un mayor grado de conductas deseables y que den mayor importancia al comportamiento deseable entre los estudiantes con TDAH.

Las formas más prominentes de conducta deseable en estos estudiantes son la disposición a asumir responsabilidades, el buen humor y el optimismo. Significativamente, también se distingue una buena disposición para llevar a cabo las tareas que se les confían (bajo la categoría de habilidades sociales básicas y gestión de sentimientos). Los estudiantes con TDAH muestran predisposición para ayudar al docente y, por último, para ayudar y colaborar con sus compañeros de clase durante el juego.

Pasando ahora a las conductas no deseables, la conducta hiperactiva más prominente en los estudiantes con TDAH es la tendencia a molestar a los compañeros de clase durante los períodos de descanso o en periodos de espera. Los consejos prácticos para el profesorado son que durante estos períodos no incluyan largos momentos de espera para realizar un ejercicio, 
que aprovechen estos momentos de espera para dar explicaciones, evitar largas reuniones para explicar una nueva situación y controlar la duración de las explicaciones.

Las conductas impulsivas más señaladas fueron interrumpir constantemente y expresar opiniones fuera de turno. Posibles medidas prácticas para mitigar o erradicar este comportamiento incluirían involucrar a los estudiantes en la explicación o en demostraciones dirigidas por el profesorado mientras la clase está atenta.

"Parece no escuchar" fue la conducta más destacada de la variable de falta de atención en los estudiantes con TDAH. Esta forma de comportamiento aparece, por ejemplo, cuando el docente está explicando una actividad o dando instrucciones a los estudiantes y posteriormente hacen algo diferente. Entre las posibles medidas prácticas, recomendamos aumentar la proximidad física (incluido el contacto físico) con los estudiantes al dar explicaciones para alentarlos a prestar mayor atención a las explicaciones del docente.

Por último, en la variable "otras formas de comportamiento", las más comunes son la desobediencia (no cuidar adecuadamente el material deportivo, no cumplir con las reglas del juego, etc.), gritar, hacer berrinches, llamar la atención y comportarse de forma disruptiva con los demás (por ejemplo, agresión física, agresión verbal y jugar de forma brusca).

El método utilizado en esta investigación permitió realizar un estudio exhaustivo del comportamiento del alumnado con TDAH en un contexto específico, la clase de EF. La identificación de los patrones de comportamiento más frecuentes del alumnado diagnosticado con TDAH en las clases de EF brinda una información valiosa para el conjunto de profesorado de EF con el fin de definir con mayor precisión sus intervenciones. A tal efecto, las investigaciones futuras tendrán como objetivo examinar las estrategias utilizadas por el profesorado de EF al tratar con alumnado con TDAH con el fin de aumentar las conductas deseables y reducir las conductas no deseables.

\section{REFERENCIAS}

ABIKOFF, Howard; JENSEN, Peter; ARNOLD, Eugene; HOZA, Betsy; HECHTMAN, Lily; POLLACK, Simcha; MARTIN, Diane; ALVIR, Jose; MARCH, John; WIGAL, Tim. Observed classroom behavior of children with ADHD: relationship to gender and comorbidity. Jounal of Abnormal Child Psychology, n. 30, p. 349-359, 2002.

AMERICAN PSYCHIATRIC ASSOCIATION (A. P. A). Diagnostic and Statistical Manual of Mental Disorders, 5 ${ }^{\text {th }}$ ed., DSM-5. Washington, DC; American Psychiatric Association, 2013.

AMERICAN PSYCHIATRIC ASSOCIATION (A.P.A.). Diagnostic and Statistical Manual of Mental Disorders: DSM-IV-TR. $4^{\text {th }}$. Washington, DC: American Psychiatric Association, 2002.

ANDERSON, Donnah L.; WATT, Susan E.; NOBLE, William; SHANLEY, Dianne C. Knowledge of attention deficit hyperactivity disorder (ADHD) and attitudes toward teaching children with ADHD: The role of teaching experience. Psychology in the Schools, v. 49, n.6, p. 511-525, 2012. 
ARAGÓN CALVO, Sonia. Comportamiento de niños TDAH en clases de Educación Física: un estudio observacional. EFDeportes.com Revista Digital, n. 115, p. 1-13, 2011. Disponible en: http://www.efdeportes.com/efd155/ninos-tdah-en-clases-de-educacion-fisica.htm. Acceso: 01 ene. 2018.

ASHWOOD, Karen L.; TYE, Charlotte; AZADI, Bahare; CARTWRIGHT, Sally; ASHERSON, Philip; BOLTON, Patrick. Brief Report: Adaptive Functioning in Children with ASD, ADHD and ASD + ADHD. Journal of Autism and Developmental Disorders, v. 45, n. 7, p. 2235-2242, 2015. Disponible en: http://doi.org/10.1007/s10803-014-2352-y. Acceso: 02 jun. 2017.

BARKLEY, Russell A. Attention-deficit hyperactivity disorder: A handbook for diagnosis and treatment. New York: Guilford, 2006.

BUNFORD, Nora; EVANS, Steven W.; WYMBS, Frances. ADHD and Emotion Dysregulation Among Children and Adolescents. Clinical Child and Family Psychology Review, v. 18, n. 3, p. 185-217, 2015. Disponible en: http://doi.org/10.1007/s10567-015-0187-5. Acceso: 02 ago. 2017.

CARRIEDO, Alejandro. Beneficios de la Educación Física en alumnos diagnosticados con Trastorno por Déficit de atención con Hiperactividad (TDAH). Journal of Sport and Health Research, v. 6, n. 1, p. 47-60, 2014.

CARSPECKEN, Phil Francis. Four Scenes for Posing the Question of Meaning and Other Explorations in Critical Philosophy And Critical Methodology. New York/Berlin: Peter Lang, 1999.

CATALÁ-LÓPEZ, Ferran; PEIRÓ, Salvadó; RIDAO, Manuel; SANFÉLIX-GIMENO, Gabriel; GĖNOVA-MALERAS, Ricard; CATALÁ, Miguel A. Prevalence of attention deficit hyperactivity disorder among children and adolescents in Spain: a systematic review and meta-analysis of epidemiological studies. BMC Psychiatry, v. 12, n. 1, p. 168, 2012. Disponible en: http://doi. org/10.1186/1471-244X-12-168. Acceso: 04 abr. 2017.

COOPER, John O.; HERON, Timothy E.; HEWARD, William L. Applied behavior analysis. $2^{\text {nd }}$ ed.. Upper Saddle River, NJ: Pearson, 2007.

EFSTRATOPOULOU, Maria A; JANSSEN, Rianne; SIMONS, Johan. Children's Deviant Behavior in Primary Education: Comparing Physical Educator's Implicit Theory With Diagnostic Criteria. Journal of Attention Disorders, 2012. Disponible en: http://doi. org/10.1177/1087054712449479. Acceso: 02 mar. 2017.

FEU, Sebastián, et al. Actitud del profesorado de educación física frente al aprendizaje cooperativo. Movimento, v. 22, n. 3, p. 929-942, 2016.

FRIEDRICHS, Bettina; IGL, Wilmar; LARSSON, Henrik; LARSSON, Jan-Olov. Coexisting Psychiatric Problems and Stressful Life Events in Adults With Symptoms of ADHD-A Large Swedish Population-Based Study of Twins. Journal of Attention Disorders, v. 16, n. 1, p. 13-22, 2012. Disponible en: http://doi.org/10.1177/1087054710376909. Acceso: 15 nov. 2017.

GOULARDINS, Juliana B.; MARQUES, Juliana C.; OLIVEIRA, Jorge A. de. Attention Deficit Hyperactivity Disorder and Motor Impairment: A Critical Review. Perceptual and motor skills, v. 124, n. 2, p. 425-440, 2017.

GUSTAFSSON, Peik; GÖRAN, Carl; ERICSSON, Ingegerd; LINDÉN, Christian; KARLSSON, Magnus K.; THERNLUND, Gunilla. Reliability and validity of the assessment of neurological 
soft-signs in children with and without attention-deficit-hyperactivity disorder. Developemntal Medicine \& Child Neurology, v. 52, p. 364-370, 2010.

KIELY, Richard; ASKHAM, Jim. Furnished imagination: The impact of pre-service teacher training on early career work in TESOL. TESOL Quarterly, v. 46, p. 496-518, 2012.

JIN, Wen-Lan; LI, Gai-Zhi; DU, Ya-Song; COGHILL, David; AU, Shun; ZHONG, Xiang-Yang. ADHD symptoms in children aged 5 to 15 years in Zhabei District, Shanghai. The European Journal of Psychiatry, v. 30, n. 3, p. 173-182, 2016.

LATORRE, Antonio. La investigación-acción: conocer y cambiar la práctica educativa. $4^{\text {th }}$ ed. Barcelona: Graó, 2007.

LAVAY, Barry; HENDERSON, Hester; FRENCH, Ron; GUTHRIE, Sharon. Behavior management instructional practices and content of college/university physical education teacher education (PETE) programs. Physical Education and Sport Pedagogy, v. 17, n. 2, p. 195-210, 2012. Disponible en: http://doi.org/10.1080/17408989.2010.548063. Acceso: 06 feb. 2018.

MAUTONE, Jennifer A.; LEFLER, Elizabeth K.; POWER, Thomas J. Promoting Family and School Success for Children With ADHD: Strengthening Relationships While Building Skills. Theory Into Practice, 2011. Disponible en: http://www.tandfonline.com/doi/abs/10.1080/00405841. 2010.534937. Acceso: 08 ago. 2017.

MCGINNIS, Ellen; GOLDSTEIN, Arnold. Programa de habilidades para la infancia temprana. La enseñanza de habilidades prosociales a los niños de preescolar y jardín infantil. Illinois: Research, 1990. Disponible en: http://www.oei.es/inicial/articulos/habilidades infancia.pdf. Acceso: 10 ago. 2017.

MERRIAM, Sharan B. Qualitative Research and Case Study Applications in Education. San Francisco: Jossey-Bass , 1998.

MORENO OLIVER, Francesc Xavier. Análisis psicopedagógico de los alumnos de Educación Secundaria Obligatoria con problemas de comportamiento en el contexto escolar.Barcelona: Universitat Autónoma de Barcelona, 2001.

O'LEARY, K. Daniel; POULUS, Rita W.; DEVINE, Vernon T. Tangible reinforces: Bonuses or bribes. Journal of Consulting and Clinical Psychology, v. 38, p, 1-8, 1972.

ORJALES, Isabel. Estrategias para la mejora de la conducta y el rendimiento escolar del niño con TDAH en el aula. 2013. Disponible en: <http://fundacioncadah.org/web/doc/index. html?id doc=92>. Acceso: 06 ago. 2017.

PATTERSON, B. R.; MC NEAL, Shirley; HAWKINS, Nancy; PHELPS, Richard. Reprogamming the social environment. Journal of Child Psychology and Psychiatry, v. 8, p. 181-195, 1967.

POLANCZYK, Guilherme V.; WILLCUTT, Erik G.; SALUM, Giovanni A.; KIELING, Christian; ROHDE, Luis A. ADHD prevalence estimates across three decades: an updated systematic review and meta-regression analysis. International journal of epidemiology, v. 43, n. 2, p. 434442, 2014.

RUIZ TRIVIÑO, Lidia. Programa para alumnado con TDAH. Revista Digital Inovación Y Experiencias Educativas, v. 27, 2010. Disponible en: http://doi.org/10.1163/ q3 SIM 00374. Acceso: 09 ago. 2017. 
SOLER PRAT, Susanna; VILANOVA SOLER, Anna. Investigacion en educación física. In: Coleccion formacion del profesorado. Educación Secundaria. Educación Física: investigación, innovación y buenas prácticas. Barcelona: Graó, 2010. p. 25-42.

SOMMER, Barbara; SOMMER, Franco. La investigación del comportamiento: una guía con técnicas y herramientas. México: Universidad Iberoamericana, 2002.

TANNOCK, Rosemary. Rethinking ADHD and LD in DSM-5: proposed changes in diagnostic criteria. Journal of Learning Disabilities, v. 46, n. 1, p. 5-25, 2013. Disponible en: http://doi. org/10.1177/0022219412464341. Acceso: 07 ene. 2018.

TANURE ALVES, Maria Luiza et al. Physical education classes and inclusion of children with disability: brazilian teachers'perspectives. Movimento, v. 23, n. 4, p. 1229-1244, 2017.

VAN DEN BAN, Els; SOUVEREIN, Patrick; MEIJER, Willemijn; VAN ENGELAND, Herman; SWAAB, Hanna; EGBERTS, Toine; HEERDINK, Eibert. Association between ADHD drug use and injuries among children and adolescents. European Child \& Adolescent Psychiatry, v. 23, n. 2, p. 95-102, 2014. Disponible en: http://doi.org/10.1007/s00787-013-0432-8. Acceso: 04 ene. 2018.

WYK, Yolanda Vand; PIENAAR, Anita E.; COETZEE, Dané. Okulêre motoriese beheer funksie en visueel-motoriese integrasie status van'n geselekteerde groep 6-tot 8-jarige leerders met ADHD. South African Journal for Research in Sport, Physical Education and Recreation, v. 34, n. 2, p. 215-226, 2012.

Apoio:

Institut Nacional d'Educació Física de Catalunya (INEFC) 